\title{
The eternal molecule
}

As a prelude to the many celebrations around the world saluting the 50th anniversary of the discovery of the DNA double helix, Nature presents a collection of overviews that celebrate the historical, scientific and cultural impacts of a revelatory molecular structure.

$\mathrm{F}$ ew molecules captivate like DNA. It enthrals scientists, inspires artists, and challenges society. It is, in every sense, a modern icon. A defining moment for DNA research was the discovery of its structure half a century ago. On 25 April 1953, in an article in Nature, James Watson and Francis Crick described the entwined embrace of two strands of deoxyribonucleic acid. In doing so, they provided the foundation for understanding molecular damage and repair, replication and inheritance of genetic material, and the diversity and evolution of species.

The broad influence of the double helix is reflected in this collection of articles. Experts from a diverse range of disciplines discuss the impact of the discovery on biology, culture, and applications ranging from medicine to nanotechnology. To help the reader fully appreciate how far the double helix has travelled, we also include the original landmark paper by Watson and Crick and the two accompanying papers by Maurice Wilkins, who shared the Nobel Prize with Watson and Crick in 1962, and by co-discoverer Rosalind Franklin, and their co-authors (pages 397-401). We are pleased to acknowledge the financial support of Roche in producing this collection of articles.

\section{Transforming science}

Given the immense significance of the double helix, it is difficult to imagine a world that wasn't transfixed by its discovery. Yet, as Robert Olby recalls on page 402, the proposed structure initially received a lukewarm reception. Maclyn McCarty, who, together with Oswald Avery and Colin MacLeod, had previously showed DNA to be the substance of inheritance, shares his personal perspective (page 406).

In science, where a lifetime's work can often be encapsulated in a few shining moments, the greatest controversies are sometimes over the sharing of credit. The discovery of the double helix is no exception. The premature death and posthumous treatment of Rosalind Franklin, whose X-ray images of DNA crystals revealed telltale clues of a double helical structure, propelled her portrayal as a feminist icon. But, as discussed here by her biographer Brenda Maddox (page 407), Franklin is better remembered as a committed and exacting scientist who saw no boundaries between everyday life and science.

Most of our readers will have grown up with the double helix, and yet it is still startling to consider how quickly DNA biology has progressed in just a lifetime. Bruce Alberts reviews how the elegant pairing of the two strands of the double helix revealed the mechanism for replicating the essential units of inheritance (page 431). Errol Friedberg considers the vulnerability of the DNA molecule to damage and the multitude of ways in which cells repair the damage (page 436). And
Gary Felsenfeld and Mark Groudine describe how the gargantuan DNA molecule is packaged inside the minuscule cells of the body, and how an additional layer of information is encrypted within the proteins intimately associated with DNA (page 448). It is perhaps salutary also to recognize what is still to be learnt about the physiological states in which DNA exists, as discussed by Philip Ball (page 421).

As reviewed by Leroy Hood and David Galas (page 444), DNA science generated the tools that spawned the biotechnology revolution. It enabled the cloning of individual genes, the sequencing of whole genomes and, with the application of computer science, transformed the nature and interactions of molecules into an information science. Carlos Bustamante and co-authors consider how we are still learning much about the distinct structural and physical properties of the molecule (page 423). And according to Nadrian Seeman, DNA may develop new applications as a material for nanoscale engineering (page 427).

\section{Influencing society}

Beyond scientific and technological forums, the double helix has imprinted on society's views of history, medicine and art. As discussed by Svante Pääbo (page 409), the records of evolution have been recalibrated with information traced through DNA sequence. On page 412, Aravinda Chakravarti and Peter Little revisit the 'nature versus nurture' debate and our developing view of the interplay between genetic and environmental factors in human disease. And DNA science will transform clinical medicine according to John Bell (page 414), providing a new taxonomy for human disease and triggering a change to health care practice. On page 440, Gustav Nossal reviews how an understanding of DNA processes, such as recombination, have transformed the field of immunology.

As a visual icon, and as a profound influence on our nature, the DNA molecule has permeated the imagery and art of our time, and is described by Martin Kemp (page 416) as the Mona Lisa of this scientific age. Given that broad impact, and revolutions that are yet to come, it is perhaps appropriate to leave the last word to an artist. Written in 1917, the poem Heredity by Thomas Hardy (see inset) seems to foreshadow both the essence and the fascination of the molecule that we celebrate here.

$\begin{array}{lr}\text { Carina Dennis } & \text { Commissioning Editor } \\ \text { Philip Campbell } & \text { Editor, Nature } \\ \text { doi:10.1038/nature } 01396 & \end{array}$

\section{Heredity \\ I am the family face; Flesh perishes, Ilive on, Projecting trait and trace Through time to times anon, And leaping from place to place Over oblivion.}

The years-heired feature that can In curve and voice and eye Despise the human span Ofdurance - that is I; The eternal thing in man, That heeds no call to die.

Thomas Hardy

(First published in Moments of Vision and Miscellaneous Verses, Macmillan, 1917) 